
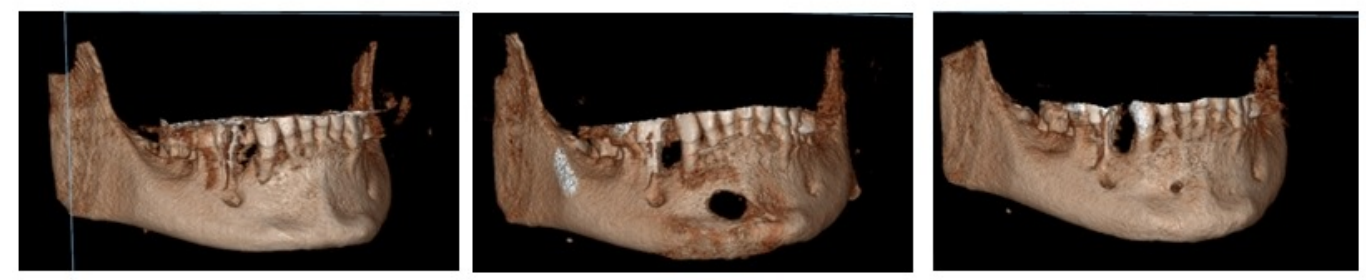

\title{
Difficult management of severe Medication-Related Osteonecrosis of the Jaws in a denosumab treated osteoporotic patient, a case report.
}

\author{
Silvia Arrigoni ${ }^{1}$, VALERIA MARTINI ${ }^{2}$, Riccardo Bonacina ${ }^{2}$ \\ 1 University of Parma \\ 2 Azienda Socio Sanitaria Territoriale (ASST) Papa Giovanni XXIII \\ Funding: The author(s) received no specific funding for this work. \\ Potential competing interests: The author(s) declared that no potential competing interests exist.
}

\section{Abstract}

In a patient treated with denosumab for osteoporosis, a case of stage II MRONJ occurred after 3 months from implant placement and subsequent implant loss. The first surgical approach led to a worsening at Stage III. A second surgery with a conservative approach was performed more than 6 months after the last denosumab injection obtaining therefore the definitive healing. Pharmacokinetics and surgical approach should be considered in MRONJ treatment scheduling.

\section{[1][2] Introduction}

Medication-related osteonecrosis of the jaws (MRONJ) is a severe adverse complication that may occur in patient treated with antiresorptive agents. Although MRONJ appears to be less frequent in patients undergoing therapies for osteomethabolic diseases, it's very important for these patients to reach a complete healing due to the life-long nature of their therapy.

\section{Case details}


A middle-aged smoker woman was referred to our unit at stage II MRONJ after 3 months from implant restoration. The patient, affected by severe osteoporosis, was first treated with Alendronate per os 70mg/week for 11 months and then switched to denosumab therapy $60 \mathrm{mg}$ sc every 6 months. The implant was placed with antibiotic treatment in the site of the first inferior right premolar tooth one month after the last denosumab injection and it was lost in 2 weeks for suppurative infection. After 3 months our visit revealed a suppurative lesion with pain irradiated to submental area, right ear, incisors and canine inferior teeth. The first surgical debridement was performed in antibiotic therapy, after 4 month from the last denosumab injection. Despite the surgical timing and the long term antibiotic therapy, the patient presented a worsening of sign and symptoms. CT scan and RMN imaging showed a progression to Stage III, with deep neck space infection (submental space involvement without fistula), severe lingual bone loss involving other 3 teeth and mandibular symphysis bone erosion. A second surgical debridement (6 months from the last denosumab injection) was performed applying a conservative approach to the teeth. The patient underwent hyperbaric support therapy (56 cycles). After 2 months the antibiotic therapy was suspended according to the clinical improvement (soft tissue healing, no fistula and no symptoms). At 1 year follow up the involved teeth don't show pathologic mobility and the CT scan confirms bone healing

\section{Conclusions}

The implant rehabilitation should be considered a possible cause of osteonecrosis also in patients undergoing antiresorptive therapy different from bisphosphonates. The surgical strategy to manage MRONJ should be based on the specific denosumab pharmacokinetic. The literature suggests that denosumab therapeutic scheme in osteoporotic patients may not be modified and surgery should be delayed for 3 months from the last administration. Implant placement must not be performed while high percentage of denosumab is still present. Adequate planning is mandatory before every kind of surgery also when treating MRONJ. High denosumab activity may cause surgical failure and worsening of symptoms. Radical surgical approach is often recommended in bisphosphonates related osteonecrosis. The different pharmacokinetic may suggest a different surgical treatment for denosumab patients. Bone healing is likely to occur when denosumab effects decrease, allowing a more conservative approach to teeth involved in MRONJ.

\section{References}

1. `John Malan, Kyle Ettinger, Erich Naumann, O. Ross Beirne. (2012). The relationship of denosumab pharmacology and osteonecrosis of the jaws. Oral Surgery, Oral Medicine, Oral Pathology and Oral Radiology, vol. 114 (6), 671-676. doi:10.1016/j.00oo.2012.08.439.

2. 'Lukas Hauer, Jan Jambura, Daniel Hrusak, Miroslava Chalupova, et al. (2020). Surgical therapy for medication-related osteonecrosis of the jaw in osteoporotic patients treated with antiresorptive agents. BIOMED PAP, vol. 164 (1), 100107. doi:10.5507/bp.2018.081. 